\section{MS12-P4 Structural basis for sensitivity of fluorescent proteins to molecular Oxygen investigated by high-pressure crystallography}

Bénédicte Lafumat ${ }^{1}$, Royant Antoine ${ }^{1,2}$, de Rosny Eve ${ }^{2}$, Van der Linden Peter ${ }^{1}$, Leonard Gordon ${ }^{1}$, Carpentier Philippe ${ }^{1}$

1. ESRF. 6 Rue Jules Horowitz, Grenoble, France

2. IBS, 6 rue Jules Horowitz, Grenoble, France

email: benedicte.lafumat@esrf.fr

Since the successful cloning of the green fluorescent protein (GFP) from the jellyfish Aequorea victoria in 1992, fluorescent proteins (FPs) have revolutionized Life Science [1]. A FP 3-dimensional structure is basically that of a $\beta$-barrel protecting the chromophore responsible for the fluorescence. FPs show scales of sensitivity to molecular Oxygen $\left(\mathrm{O}_{2}\right)$ that is most likely related to the porosity of the $\beta$-barrel combined with the reactivity of the chromophore. In particular, photosensitizing FPs [2] consume $\mathrm{O}_{2}$ and generate cytotoxic reactive oxygen species (ROSS) upon photobleaching and are thus of interest for light-induced cell killing methods [3]. Our project aims at studying the flexibility/porosity of $\beta$-barrels and the reactivity of chromophores, and thus at comprehending the mechanism that govern the sensitivity of FPs to $\mathrm{O}_{2}$. We are exploiting pressure and high-pressure crystallography to explore the conformational sub-states and reveal the dynamic motions of $\beta$-barrel strands of FPs, and secondly to indentify $\mathrm{O}_{2}$ pathway and specific binding site in FPs. Specific cells have been designed to cryo-cool protein crystals under pressure or high-pressure of Helium (He) [4], Krypton ( $\mathrm{Kr})$, and $\mathrm{O}$ gas. Indeed, it has been shown that high-pressure crystallography with the He cryo-cells allows exploring the energy landscapes of proteins, providing access to their conformational sub-states and their dynamics [2]. $\mathrm{O}$, and $\mathrm{Kr}$ cryo-cells allow populating channels and potential sites of affinity for $\mathrm{O}_{2}$ in FPs. In complement to X-ray crystallography, pressure induced modifications of PFs are characterized by in-crystallo UV-visible and Raman spectroscopy. For these studies, a set of FPs have been produced and crystallized including the efficient photosensitizer KillerRed. During this presentation, we will introduce the different methodologies and present the pressure-induced structural changes of some FPs, which already allows us to indentify flexible weakness in $\beta$-barrels and potential entrance/exit gates for $\mathrm{O}_{2}$ in FPs. The ultimate purpose of this work will be to provide the structural basis that allows identifying efficient photosensitizers that could have important applications in cancer photodynamic therapy.

[1] Shimomura et al (1962) J Cell Comp Physiol. 59, 223-39; [2] Carpentier et al (2012) J. Am. Chem. Soc. 134, 18015-21; [3] Jacobson et al (2009), Trends in Cell Biology. 18 (9), 443-50;[4] Van der Linden et al (2014) J. Appl. Cryst. 47, 584-92

Keywords: Fluorescent proteins, molecular Oxygen, Photosensitizer, High pressure, X-ray crystallography
MS12-P5 The feasibility of crystallization of flexible proteins using maltose-binding protein

Yuya Hanazono $^{1}$, Kazuki Takeda ${ }^{1}$, Kunio Miki ${ }^{1}$

1. Graduate School of Science, Kyoto University

email: yuya-h@kuchem.kyoto-u.ac.jp

Sufficient quality crystals of proteins are indispensable to determination of the whole protein structures including the side chains by using X-ray crystallography. It has been reported that protein tags, such as maltose-binding protein (MBP), glutathione S-transferase, thioredoxin are useful to get better crystals. Though previous crystallographic studies through the use of fusion protein were carried out to reveal the structure for rigid proteins or domains, there are many flexible proteins or peptides, which are required further researches. In order to examine the feasibility of crystallization of flexible proteins or peptides using rigid protein tags, we studied about the two such examples where flexible peptides are fused with MBP.

We determined two structures of flexible peptides fused with MBP. Crystals of the peptides with MBP diffracted $\mathrm{X}$-rays at $2 \AA$. In their crystal structures, these peptides whose electron densities are well defined have secondary structures. CD spectroscopic analysis indicated that the secondary structure contents in the crystals are comparable with those in the $30 \%$ TFE solution, which simulates in vivo conditions. These results show that the crystal structures represent the structures of the peptides in the cells. Therefore, crystallographic approach fusing with rigid protein tags could be a promising tool to determine the structure of the flexible peptides.

Keywords: maltose-binding protein, flexible peptide, circular dichroism 